\title{
Layered video multicast using fractional frequency reuse over wireless relay networks
}

\author{
Chung-Nan Lee*, Ying-Tsuen Chen, Yung-Cheng Kao, Hui-Hsiang Kao and Steve Haga
}

\begin{abstract}
Nowadays, multimedia services over wireless networks are increasingly popular. With multicast, many mobile stations can join the same video group and share the same radio resource to efficiently increase frequency utilization. However, users may be located at different positions, and so suffer different degrees of path loss and interference, and receive a different signal-to-interference and noise ratio (SINR). Users at the cell-edge receiving a lower SINR may degrade the multicast efficiency. In this article, we propose four schemes that consider fractional frequency reuse (FFR) over relay networks in multi-cells. With FFR, users close to a base station (BS) are given more resources to improve the video quality. An efficient resource allocation scheme is also proposed. Compared to the conventional relay scheme, the proposed schemes can provide over $10 \%$ more video layers for all users and give better video quality for users near the BS.
\end{abstract}

Keywords: wireless relay networks, multicast, layered video multicast, resource allocation, fractional frequency reuse

\section{Introduction}

In coming years, the growing use of multimedia services and streaming, such as live TV and on-demand video, is expected to be one of the most attractive services of wireless networks. Compared to wired networks, wireless networks have a limited and more expensive bandwidth. Therefore, in order to efficiently utilize wireless resources, users accessing the same media program can join the same multicast group and be serviced simultaneously. As users within the same multicast group are located at different positions and experience various levels of path loss, they may receive a different signalto-interference and noise ratio (SINR). For a high probability of correctly decoding video data transmitted from the base station (BS), a user requires a sufficient SINR. Traditionally, a lower transmission rate is needed to support a satisfactory multicast service for all users in the same group. Therefore, users at the cell-edge who suffer a larger path loss can only receive a lower SINR, resulting in poor multicast efficiency.

In order to provide better coverage and throughput, relay technology has been considered in various standards (e.g., IEEE 802.16j, IEEE $802.16 \mathrm{~m}$, and LTE-Advanced). In

\footnotetext{
* Correspondence: cnlee@cse.nsysu.edu.tw

Department of Computer Science and Engineering, National Sun Yat-sen University, Kaohsiung, Taiwan
}

addition to increasing the number of BSs, low-cost and easy-placement relay stations (RSs) are also an efficient solution for improving the performance of cell-edge areas. As shown in Figure 1, the RSs are located between the BS and the users. The transmission is divided into two steps. In the first step, the BS transmits data to the RSs and users with good SINR. Then, in the second step, the RSs transmit data to users that are far from the BS and unable to receive data in the first step. For users in the cell-edge area, the SINR of BS RS and RS user connection would be better than that of BS user connection, so this two-step process can use a higher transmission rate and reduce the difference in SINR among users in the service area [1]. RSs also increase the potential for reuse by taking into account spatial diversity and channel diversity [2].

The reuse factor $(K)$ of a network is defined as the number of adjacent cells operating on different frequencies, i.e., each $K$ adjacent cells operate on separate frequencies. In other words, each cell uses $1 / K$ of the total frequency. In conventional OFDMA-based wireless networks, as shown in Figure 2a, each cell operates on a different frequency from its neighbors to prevent interference between cells. Thus, a $K$ value of greater than one is obtained, which causes poor frequency utilization. Lower values of $K$ reuse more frequencies, but have a higher interference. For better frequency utilization, a reuse factor equal to one was 


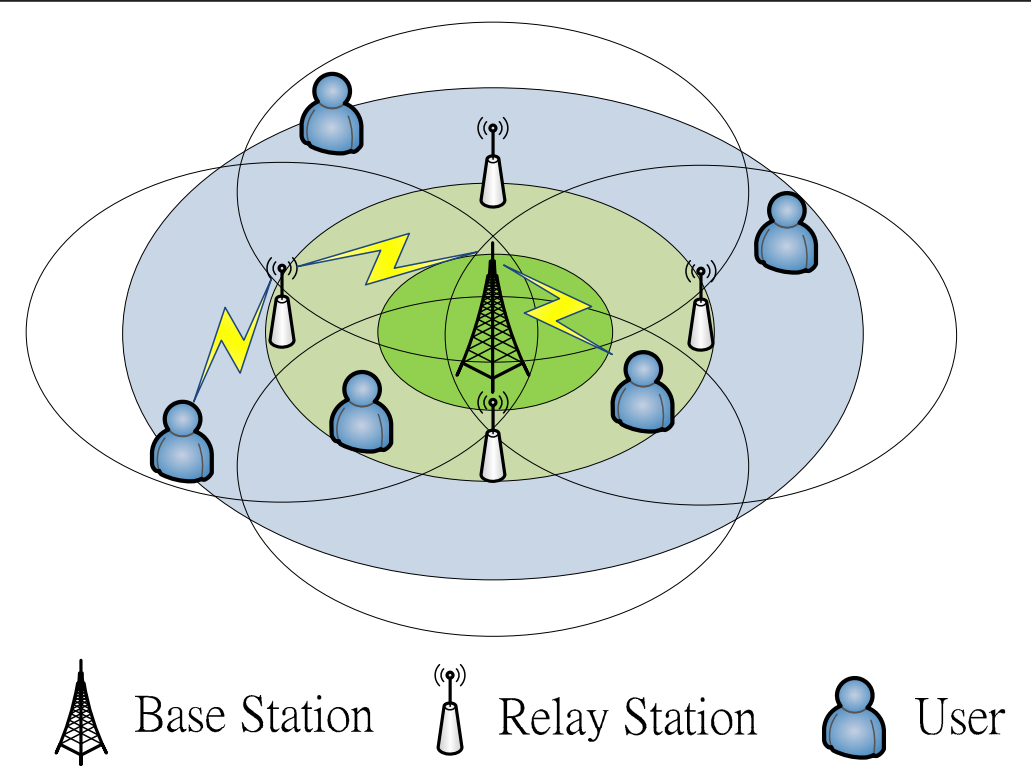

Figure 1 Relay networks.

considered for the fractional frequency reuse (FFR) studied in [3-6]. As shown in Figure 2b,c, the total frequency is divided into three equal partitions: F1, F2, and F3. The unicast users are divided into groups of inner users and outer users. The BS can use part of the frequency (e.g., F2 + F3) to serve the inner users, and the other part of the frequency (e.g., F1) for the outer users. The inner users may suffer co-channel interference (CCI) from neighboring cells, but this scheme can use more frequencies than the conventional scheme.

In addition, scalable video coding (SVC) $[7,8]$ can efficiently guarantee different video quality for users with heterogeneous bandwidth. By applying SVC, the original video is encoded into one base layer and several enhancement layers. Users with a poor SINR can receive fewer layers, while those with better SINR can receive more layers to enjoy better video quality.

Recent research into relay communications can be found in $[2,9,10]$. Jin and $\mathrm{Li}[2]$ exploited channel diversity for different users, studying cooperative multicast transmission combined with network coding to improve performance. Hou et al. [9] proposed a multicast scheduling scheme with user cooperation to achieve high performance and maintain fairness across groups. Elrabiei and Habaebi [10] presented a power-efficient cooperative transmission scheme that considers relay selection to significantly minimize the number of cooperative users and reduce power consumption. However, the IEEE 802.16j

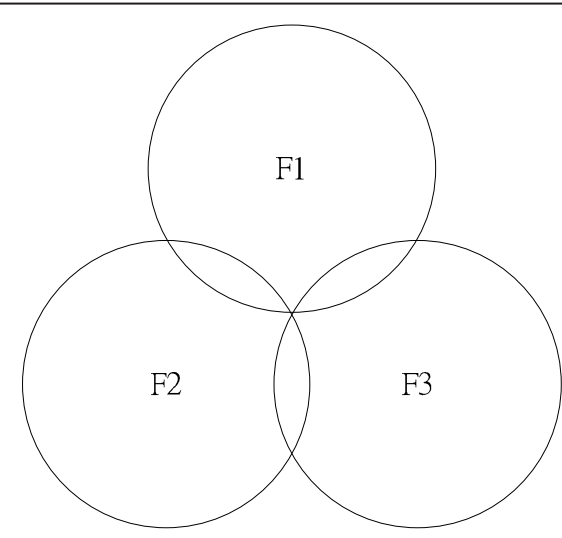

(a)

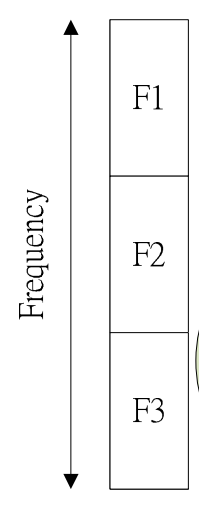

(b)

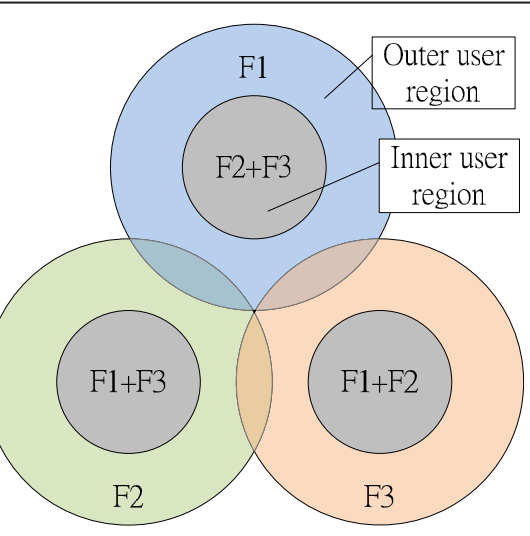

(c)

Figure 2 Comparison of frequency planning: (a) Conventional reuse scheme. (b) Frequency band. (c) FFR scheme. 
standard specifies a direct connection between the BS and users, or a connection between the BS and users with the help of RSs, but does not allow direct communication among users [11]. Therefore, the studies $[2,9,11]$ require some modifications before it can be applied to a scenario with specified RSs.

Zhao and Su [12] analyzed the performance of cooperative and relay transmissions, and derived a closedform formulation to model the average outage probabilities. Using this formulation, optimal cooperation strategies for the relay location and power allocation are provided. Multicast routing in relay networks has been investigated in $[13,14]$, where the protocol to find an appropriate routing path to increase the system performance was proposed. Sheen et al. [15] investigated optimal RS placement, path selection, resource allocation, and frequency reuse. However, only a few works consider multicasts over relay networks.

Kuo and Lee [16] proposed a multicast recipient maximization scheme to achieve near-optimal solutions. Research into video multicast scheduling over wireless relay networks was presented in $[1,17]$. Alay et al. [1] considered both omni-directional and directional relays to analyze the optimal system parameters for maximizing the video quality, and $\mathrm{Yu}$ et al. [17] proposed a utility-based algorithm to maximize the system utility for scalable video multicasts. Different from [1,17], the proposed schemes in this article increase frequency utilization by considering FFR in multi-cells.

In the proposed schemes, we define groups of inner users and outer users. Inner users are served by the BS with a lower reuse factor, and outer users are served by the RSs with a higher reuse factor. For example, as shown in Figure 3a,b, in the first step, the BS uses the whole frequency $(K=1)$, before the RSs use $K=3$ to serve outer users in the second step. In the conventional relay scheme, as shown in Figure $3 \mathrm{c}$, d, both steps use a reuse factor of 3 to avoid inter-cell interference. With FFR, the BS has more resources available in the first step, and thus requires a shorter transmission time than the conventional scheme to transmit the same video layers, and the RSs have a longer transmission time in the second step. Therefore, the number of video layers that RSs can transmit in the second step is mainly limited by the available resources, so outer users can receive more video layers than with the conventional scheme.

In this article, the proposed schemes using frequency reuse in multi-cells have more efficient frequency utilization than the conventional scheme. With frequency reuse, the BS can use additional frequencies to transmit more video layers to inner users. In order to use frequency efficiently, a resource allocation scheme is proposed to provide as many video layers as possible to all users while keeping a satisfactory service, and to provide additional video layers to inner users who have a good SINR. Compared to the conventional scheme, the proposed schemes provide better video quality for cell-edge users and provide more video layers to all users.

The rest of this article is organized as follows. Section 2 introduces the background to FFR and related study. A description of the problem and the proposed schemes is given in Section 3, and the results of simulations are presented in Section 4. Finally, conclusions are drawn in Section 5.

\section{Background and related study}

\subsection{Overview of FFR}

Conventionally, FFR is used to increase frequency utilization efficiency and reduce interference. There are two main FFR types, hard and soft, which we now describe. The main difference is that soft FFR reuses more frequency for inner users, such that the BS needs to lower its transmission power to avoid interfering with users of neighboring cells.

(1) Hard FFR: As shown in Figure 4, the BS transmits data to inner users by inner band F1, and outer users share the outer bands F2, F3, and F4 with outer users in neighboring cells. For example, these BSs use F1 to transmit data to inner users, and use F2, F3, and F4 to transmit data to outer users. In cell $\mathrm{A}$, outer users receiving data through $\mathrm{F} 2$ have no interference from neighboring cells; only inner users could suffer interference through F1 from neighboring cells. Once inner users are close to one BS (and far from neighboring BSs), the SINR degradation caused by the interference from neighboring BSs is acceptable.

(2) Soft FFR: As shown in Figure 5, each BS uses a part of the frequency (e.g., F1 for cell A, F2 for cell B, and F3 for cell C) to serve outer users, and uses the other part of the frequency (e.g., F2 + F3 for cell A, F1 + F3 for cell B, and F1 + F2 for cell C) to serve inner users. Thus, a reuse factor $K=3$ is applied to outer users in each cell, and whole frequencies $(K=1)$ are used to serve inner users. In soft FFR, outer users would suffer interference from neighboring cells. To avoid causing serious interference, each BS should use a lower transmission power for inner users. For example, the BS in cell A uses F1 to serve outer users, and the BSs in cell B and cell C use F1 + F3 and F1 + F2, respectively, to serve inner users, so that will cause interference due to the simultaneous use of F1. As cell-edge users in cell A, who are far from their BS and close to the neighboring BSs, receive data through F1, the interference from the use of frequency F1 in cells B and C cannot be neglected. To reduce the interference suffered by celledge users in cell A, the BSs in cells B and C must use a lower transmission power for inner users. 


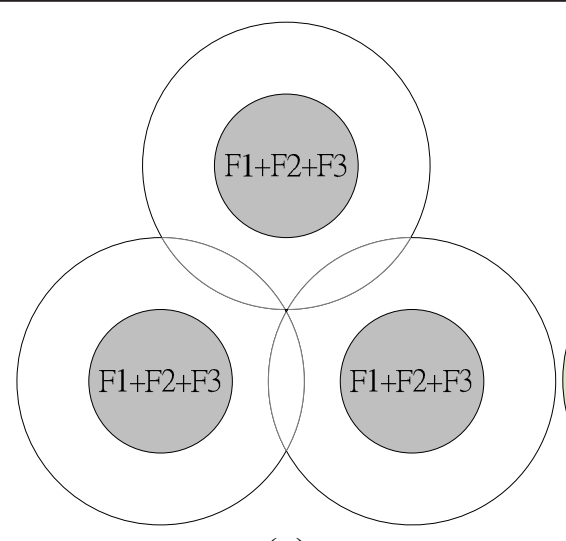

(a)

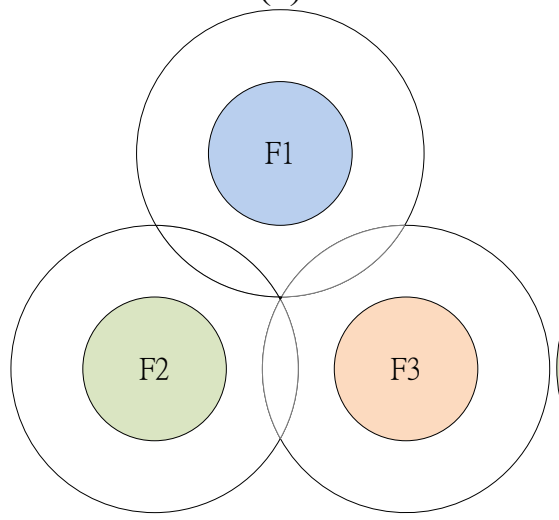

(c)
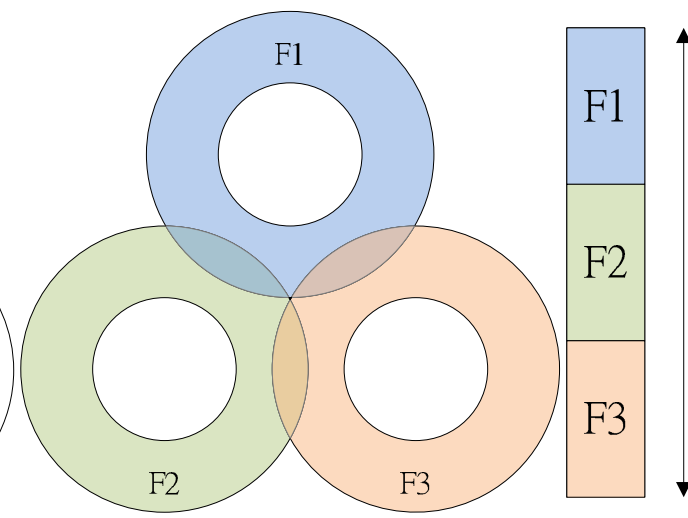

(b)

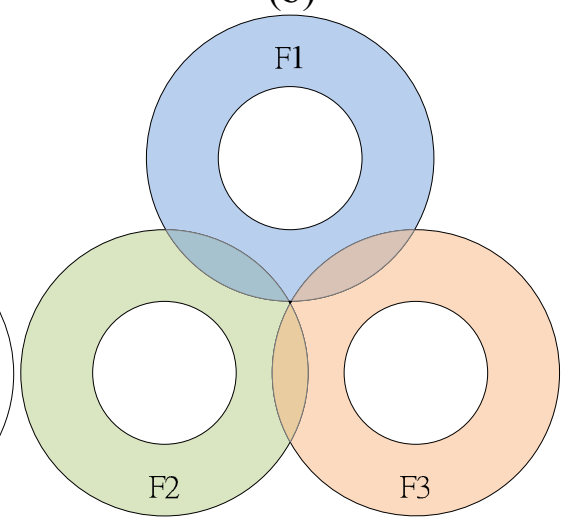

(d)

Figure 3 Comparison of frequency planning: (a) First step of the proposed scheme. (b) Second step of the proposed scheme. (c) First step of the conventional scheme. (d) Second step of the conventional scheme.

\subsection{Adaptive modulation and coding}

To achieve a transmission rate close to the theoretical channel capacity, adaptive modulation is used to adjust different modulation schemes according to users' channel conditions. Both LTE-advanced and WiMAX support these modulation levels [18,19], which are quadrature phase shift keying (QPSK), 16-state quadrature amplitude modulation (16-QAM), and 64-state QAM (64-QAM). Several channel coding schemes are supported. For example, in IEEE 802.16 [19], OFDMA architecture supports Convolution Code (CC), Block Turbo Code (BTC), Convolution Turbo Code (CTC),
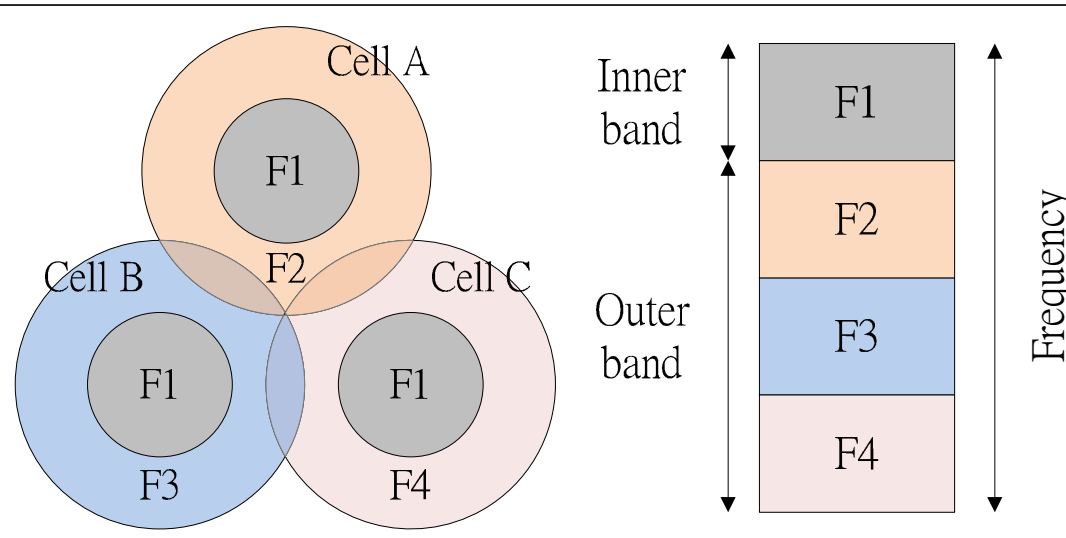

Figure 4 Hard FFR. 


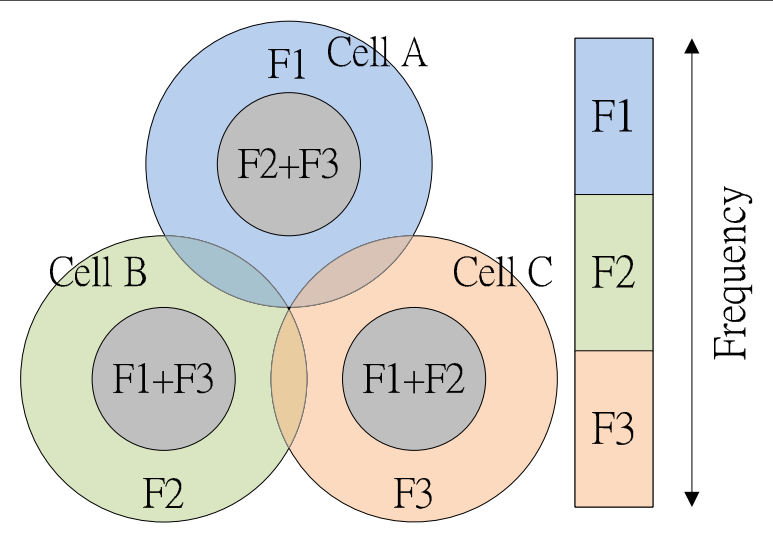

Figure 5 Soft FFR.

and Low Density Parity Check Code (LDPCC). BTC and CTC are optional. Channel coding can improve the error correction capability, but entails an additional delay and complex decoding. When a user's SINR is good, the modulation and coding scheme (MCS) with the highest transmission rate is used. Different modulation and coding levels would then be dynamically adjusted based on the user's channel condition. Each user measures channel state information and periodically feeds the information back to the BS, allowing the BS to select a suitable MCS for each user.

\subsection{Channel model}

To calculate the SINR that a user can receive, the channel model in [6,20] is applied as follows:

$$
\operatorname{SINR}_{k}=10 \cdot \log _{10}\left(\frac{\mathrm{Pt}_{i, c} / \mathrm{PL}_{i k, c}}{\text { Noise }_{c}+\sum_{a \in W} \mathrm{Pt}_{a, c} / \mathrm{PL}_{a k, c}}\right)
$$

The transmission power of BS (or RS) $i$ on a set of channels $c$ is denoted by $\mathrm{Pt}_{\mathrm{i}, \mathrm{c}}$. $\mathrm{PL}_{\mathrm{ik}, \mathrm{c}}$ denotes the path loss, calculated using the COST 231 Hata modeling [20], from station $i$ to user $k . \sum_{\mathrm{a} \in \mathrm{W}} \mathrm{Pt}_{\mathrm{a}, \mathrm{c}} / \mathrm{PL}_{\mathrm{ak}, \mathrm{c}}$ is the sum of the interference from all stations. As shown in Figure $6, W$ is the set of BSs and RSs in neighboring cells. Noise $_{c}$ denotes the noise level of the set of channels that are being used, and the same value is assigned for all users and all channels. To simplify the channel model, the transmission power is evenly distributed to all channels and the cooperative transmission gain is not considered. As illustrated in Figure 6a, we assume that the main interference in a cell comes from six neighboring cells, while that from other cells is negligible. Figure $6 \mathrm{~b}$ shows that each cell has six RSs. Outer users in the central cell receive data from an RS through frequency F3 (Figure 6a), so they suffer interference from the six neighboring cells due to the BSs in cells A, $\mathrm{C}$, and E using frequency F2 + F3, and the BSs in cells
$\mathrm{B}, \mathrm{D}$, and F using frequency F1 + F3. As the transmission power is evenly distributed to all channels, each neighboring BS transmits frequency F3 at half power.

\section{Proposed schemes}

\subsection{FFR schemes with relay}

The proposed schemes are suitable for multiple BSs. To simplify the system environment, we consider a central cell and six neighboring cells, as shown in Figure 6a. Each cell has six RSs, as shown in Figure 6b. We also assume that these systems operate in half duplex, i.e., RSs cannot receive and transmit data simultaneously. Several researchers [9], [21-23] have presented that a synchronization assumption is feasible. In this article, we assume that all RSs in a cell can synchronize their transmissions perfectly, so that synchronization errors are negligible. RSs in a cell can multicast data simultaneously without causing co-channel interference and degrading users' SINR. Cooperative transmission gain is not considered in this article. We apply FFR to two steps of multicast transmission. In the first step, the BS transmits video layers to inner users and RSs, and in the second step, all RSs simultaneously transmit video layers to outer users. With frequency reuse, the BS can transmit additional video layers to inner users. The four proposed FFR schemes are described as follows.

(1) Hard FFR with relay: In the first step, the BS in cell A can use both the inner band (F1) and the outer band (F2) to transmit data to inner users and RSs, as shown in Figure 7a. As inner users receiving data from the BS in cell A through inner band F1 suffer interference from the BSs in cells $B$ and $C$, only inner users who are close to the BS and have a good SINR can receive data successfully. The inner users receiving data through the outer band (F2) have no interference and, consequently, a better SINR. In the second step, RSs in cell A use F2 to transmit data to outer users, and the BS uses F1 to transmit additional video layers to inner 


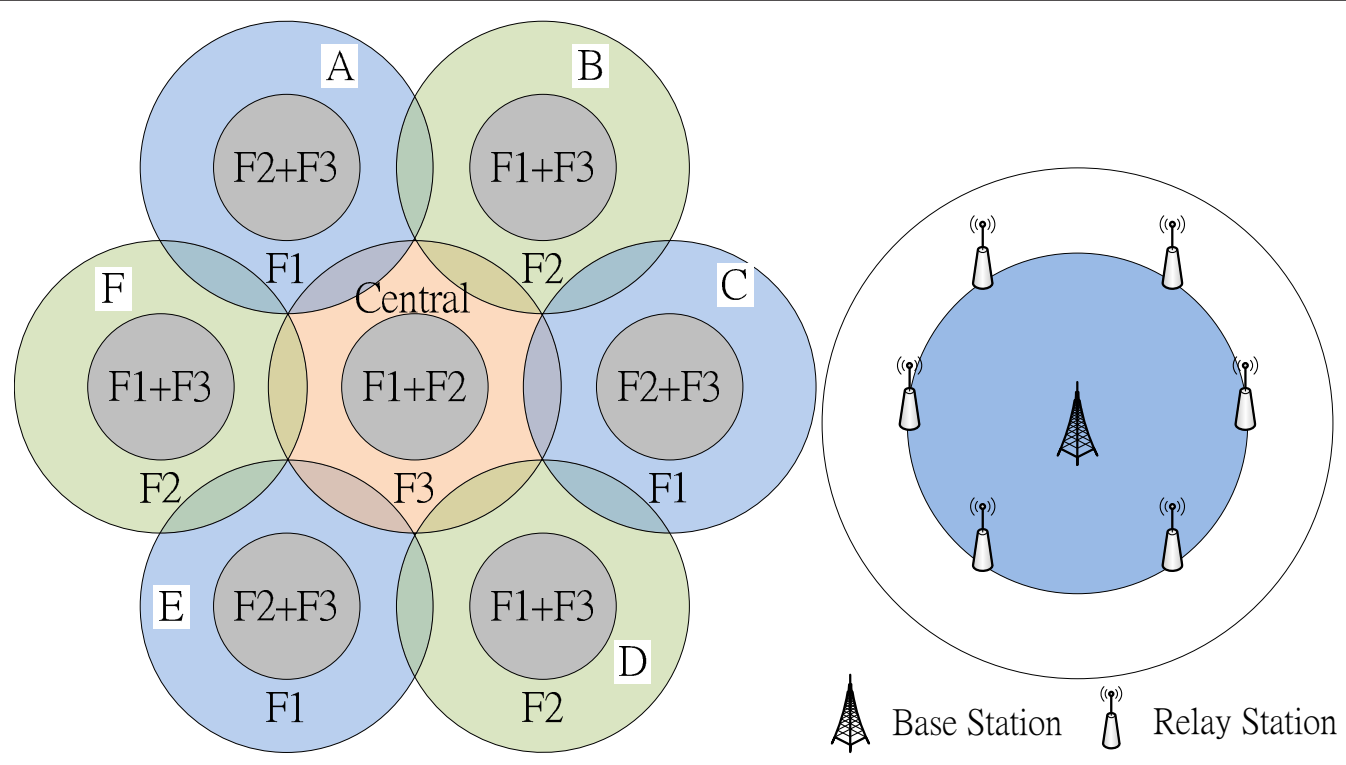

Figure 6 An example of cell planning: (a) An example of system interference. (b) Model of the relay network.

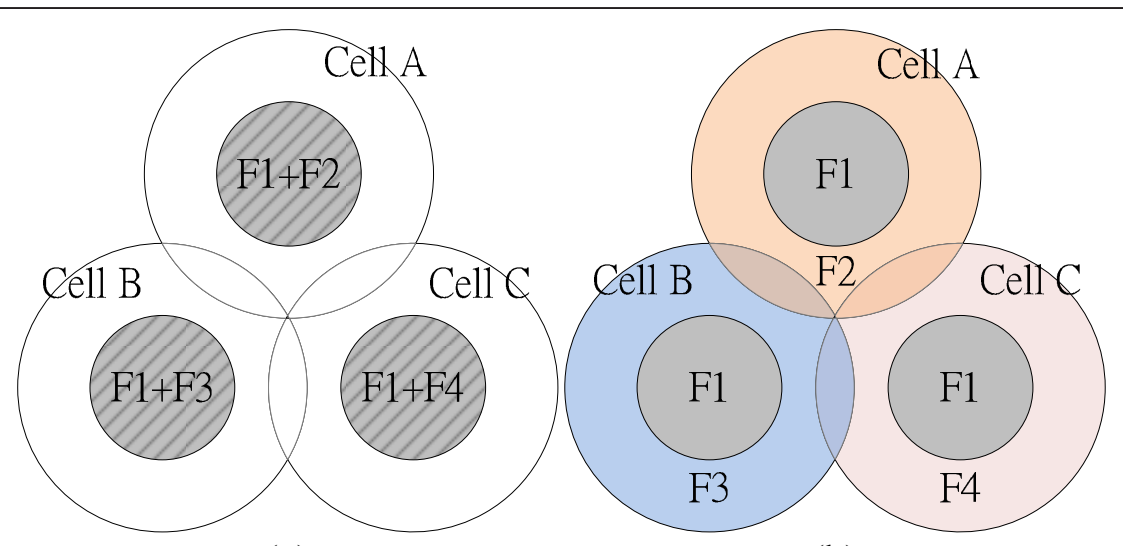

(a)

(b)

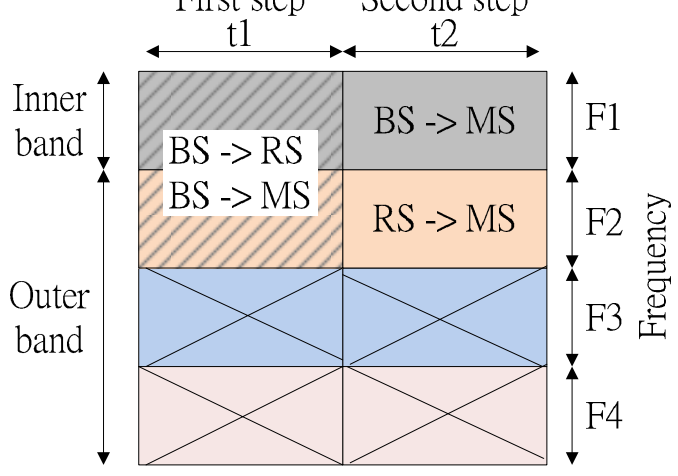

(c)

Figure 7 Frequency planning of Hard FFR: (a) First step. (b) Second step. (c) Radio map of cell A. 
users at the same time, as shown in Figure 7b. In cell A, outer users receive data from RSs through the outer band (F2) with no interference. Although the BSs in cells $B$ and $C$ cause interference on the inner band, inner users in cell A who have a good SINR can still receive data successfully. The radio map of cell A is shown in Figure 7c.

(2) Traditional FFR with relay: In the first step, the BS can use the whole frequency (F1 + F2 + F3) to transmit data, as shown in Figure 8a. Due to the interference from neighboring cells, only inner users can receive data. In the second step, as for the conventional scheme, RSs in each cell share a frequency with RSs in neighboring cells. The RSs of cells A, B, and C use F1, F2, and F3, respectively, and the BSs do not transmit anything to prevent causing interference for cell-edge users in neighboring cells, as shown in Figure 8b. The radio map of cell A is shown in Figure 8c. To avoid the interference caused by RSs and neighboring BSs transmitting at the same time, neighboring cells must synchronize the transmission time of the two steps. For example, when neighboring cells are not synchronized, the BS in cell A uses the whole frequency (F1 + F2 + F3) in its first step at the same time as the RSs in cell B are using F2 in their second step. The cell-edge users in cell B that suffer interference due to the BS of cell A thus receive a poor SINR. Therefore, each cell cannot dynamically adjust its transmission time independently.

(3) Soft FFR with relay: Similar to the first step of the Traditional FFR, the BS can use the whole frequency range, as shown in Figure 9a. In order to increase frequency utilization, the second step differs from that of the Traditional FFR scheme. The BS reuses the remaining frequency in the second step to transmit additional video layers to inner users, as shown in Figure 9b. As described in Section 2.1, to prevent serious interference with the RS transmissions of neighboring cells, the BS needs to lower its transmission power. Due to this lowered transmission power in both steps, synchronization with neighboring cells is not necessary. The radio map of cell A is shown in Figure 9c.

(4) Two-step power soft FFR with relay: This differs from the Soft FFR scheme in that, in order to increase the signal strength for inner users in the first step, the BS uses its original transmission power before lowering the power level in the second step. Similar to the

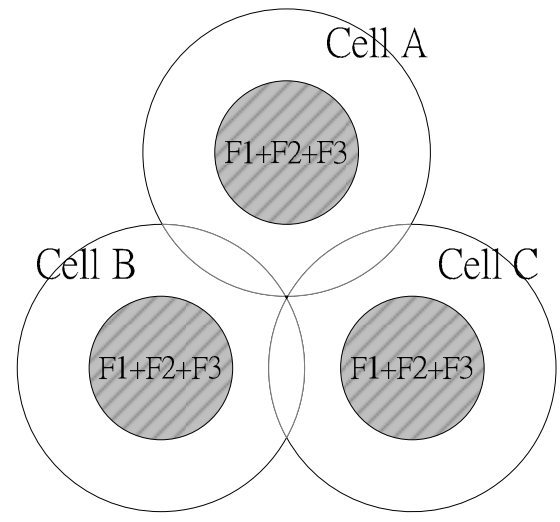

(a)

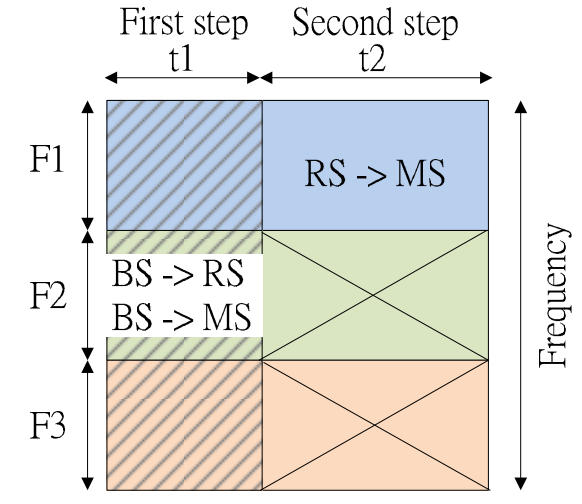

(c)

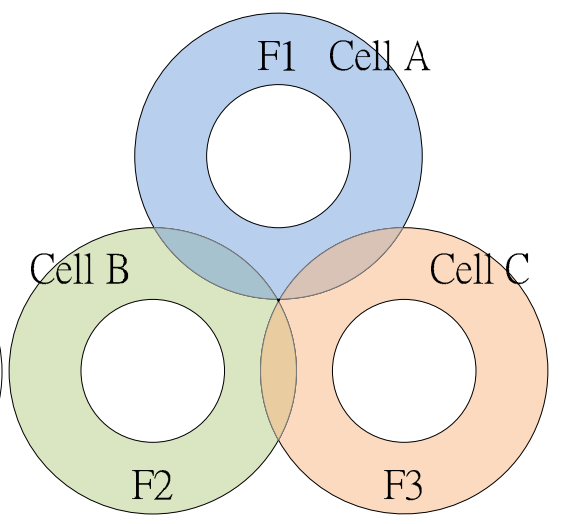

(b)

Figure 8 Frequency planning of Traditional FFR: (a) First step. (b) Second step. (c) Radio map of cell A. 


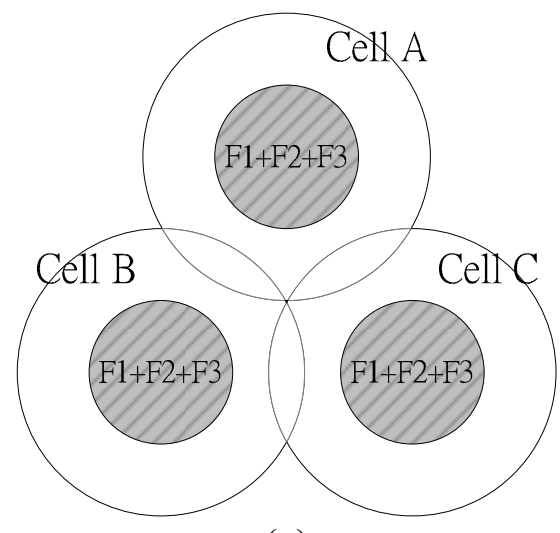

(a)

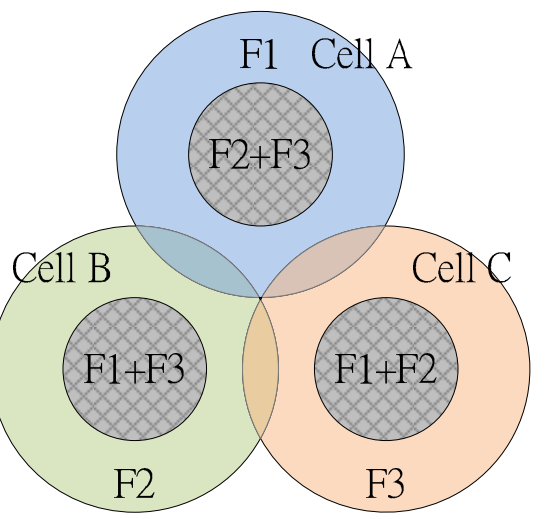

(b)

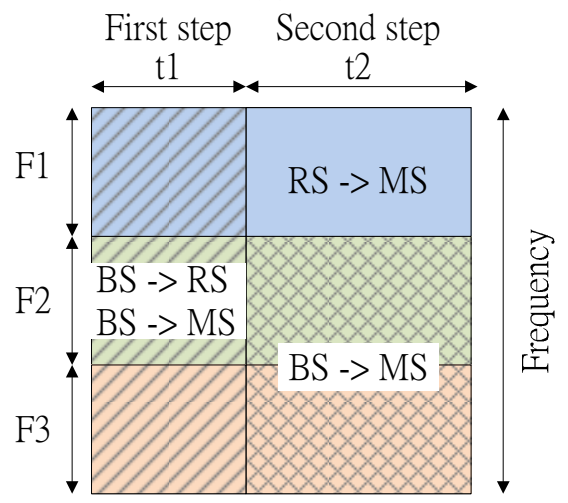

(c)

Figure 9 Frequency planning of Soft FFR: (a) First step. (b) Second step. (c) Radio map of cell A.

Traditional FFR, this scheme must synchronize its transmission time with neighboring cells. Compared to the Soft FFR, the Two-step power soft FFR provides a better SINR to inner users in the first step, but the transmission time cannot be changed dynamically.

The main points of the four FFR schemes described above are summarized in Table 1.

\subsection{Resource allocation scheme}

The goals of the proposed resource allocation scheme can be described as follows. The scheme should provide video layers as satisfactorily as possible to all users. The inner users with good SINRs should be allowed to receive more video layers. Moreover, in order to maintain fairness between groups, the scheme should allocate the limited resources according to each group's service condition. For example, assume that there are three multicast groups and the resources are limited. We denote $\{x, y, z\}$ as the number of video layers that are provided to cell-edge users of the three different multicast groups ( $x$ layers for cell-edge users in the first group, $y$ layers for cell-edge users in the second group, and $z$ layers for cell-edge users in the third group). Assume that two different assignments, $\{2,2,2\}$ and $\{1,3,3\}$, can be allocated to cell-edge users of these three groups. Although the assignment $\{1,3,3\}$ provides a total of seven layers, the six layers of the $\{2,2,2\}$ assignment should be selected in order to maintain fairness. The notation used in this section is given in Table 2 .

Table 1 Main differences among the four FFR schemes

\begin{tabular}{|c|c|c|c|}
\hline \multirow[t]{2}{*}{ FFR scheme } & \multirow[t]{2}{*}{ Synchronization among neighboring cells } & \multicolumn{2}{|c|}{ BS power } \\
\hline & & Step 1 & Step 2 \\
\hline Hard & No & Normal & Normal \\
\hline Traditional & Yes & Normal & None \\
\hline Soft & No & Low & Low \\
\hline Two-step power soft & Yes & Low & Normal \\
\hline
\end{tabular}


Table 2 List of notation

\begin{tabular}{|c|c|}
\hline Notation & Description \\
\hline V & All videos played in multicast groups \\
\hline L & Number of video layers a video is encoded in \\
\hline$x$ & Index of a video layer $\{1,2, \ldots, L\}$ \\
\hline$v_{x}$ & $x$ th layer of video $v$ \\
\hline$N_{v}$ & Users request video $\mathrm{V}$ \\
\hline$m_{1}^{v, x}$ & MCS used by the BS for the $x$ th layer of video $v$ in the first step \\
\hline$m_{2}^{v, x}$ & MCS used by the RSs for the $x$ th layer of video $v$ in the second step \\
\hline$m_{3}^{v, x}$ & MCS used by the BS for the $x$ th layer of video $v$ in the second step \\
\hline$R\left(m_{j}^{v, x}\right)$ & Transmission rate of the MCS (bits/slot) \\
\hline$T$ & Total multicast time \\
\hline$t 1$ & Duration of the first step \\
\hline t2 & Duration of the second step \\
\hline$S_{1}$ & Total resources available in the first step (slot) \\
\hline$S_{2}$ & Resources available for the RSs in the second step (slot) \\
\hline$S_{3}$ & Resources available for the BS in the second step (slot) \\
\hline bit $_{v, x}$ & Bit rate of the $x^{\text {th }}$ layer of video $v$ (bits/OFDMA frame) \\
\hline$S_{1}^{\text {used }}$ & Total resources used in the first step (slot) \\
\hline$S_{2}^{\text {used }}$ & Resources used by the RSs in the second step (slot) \\
\hline$S_{3}^{\text {used }}$ & Resources used by the BS in the second step (slot) \\
\hline B & Total frequency band in the system (subchannel) \\
\hline$C_{1}$ & Resources available for the BS in the first step within a basic service time \\
\hline$C_{2}$ & Resources available for RSs in the second step within a basic service time \\
\hline
\end{tabular}

The available frequencies for the different FFR schemes described in Section 3.1 are summarized in Table 3. B denotes the total frequency band of the system. When the subcarrier permutation is in the partial usage of subchannels (PUSC) mode and the FFT size is 1024, a slot (the basic resource element) contains two subchannels and the total number of subchannels is 30. For Hard FFR, as shown in Figure 7c, the number of subchannels F1 is set to six and F2 is set to eight to balance the available resources for the BS and RSs. As shown in Figures $8 \mathrm{c}$ and $9 \mathrm{c}$, the RSs can use ten subchannels for Traditional FFR, Soft FFR, and Two-step power soft FFR. The symbol $C_{1}$ denotes the available resources for the $\mathrm{BS}$ in the first step within a basic service time, and $C_{2}$ denotes the available resources for the RSs in the second step within a basic service time. $C_{1}$ and $C_{2}$ are calculated as follows for the Hard and Soft FFR.
Hard FFR: $C_{1}=14 / 30 \times B, C_{2}=8 / 30 \times B$.

Soft FFR: $C_{1}=B, C_{2}=1 / 3 \times B$.

The total multicast time in a frame is set to $T$. The transmission times in the first and second steps are $t 1$ and $t 2$, and thus $T=t 1+t 2$. The symbol $S_{1}$ denotes the total available resources for the BS in the first step, $S_{2}$ denotes the available resources for the RSs in the second step, and $S_{3}$ denotes the additional resources for the BS in the second step. A comparison of the available resources is listed in Table 4. Although the transmission times for the Hard and Soft FFRs are not predetermined, in order to compare the available resources of the two steps, the available resource for the BS in the first step $\left(S_{1}\right)$ is set to equal the available resource for the RSs in the second step $\left(S_{2}\right)$ in Table 4 . Thus, the transmission time ratio of the two steps is set to $1: 3$ for the Traditional FFR, the Soft FFR, and the Two-step power soft FFR.

Table 3 Comparison of available frequencies

\begin{tabular}{llllll}
\hline & Conventional relay & Hard FFR & Traditional FFR & Soft FFR & Two-step power soft FFR \\
\hline Available frequencies for BS in step 1 & $F 1$ & $F 1+F 2$ & $F 1+F 2+F 3$ & $F 1+F 2+F 3$ & $F 1+F 2+F 3$ \\
& $=10 / 30 \times B$ & $=14 / 30 \times B$ & $=30 / 30 \times B$ & $=30 / 30 \times B$ & $=30 / 30 \times B$ \\
Available frequencies for RS in step 2 & $F 1$ & $F 2$ & $F 1$ & $F 1$ & $F 1$ \\
& $=10 / 30 \times B$ & $=8 / 30 \times B$ & $=10 / 30 \times B$ & $=10 / 30 \times B$ & $=10 / 30 \times B$ \\
Available frequencies for BS in step 2 & 0 & $F 1$ & 0 & $F 2+F 3$ & $F 2+F 3$ \\
& & $=6 / 30 \times B$ & & $=20 / 30 \times B$ & $=20 / 30 \times B$ \\
\hline
\end{tabular}


Table 4 Comparison of available resources

\begin{tabular}{llllll}
\hline & Conventional relay & Hard FFR & Traditional FFR & Soft FFR & Two-step power soft FFR \\
\hline $\mathrm{S}_{1}$ & $1 / 3 \times B \times \mathrm{t} 1$ & $14 / 30 \times B \times \mathrm{t} 1$ & $B \times \mathrm{t} 1$ & $B \times \mathrm{t} 1$ & $B \times \mathrm{t} 1$ \\
& $=1 / 6 \times B \times T$ & $\cong 5.09 / 30 \times B \times T$ & $=1 / 4 \times B \times T$ & $=1 / 4 \times B \times T$ & $=1 / 4 \times B \times T$ \\
$\mathrm{~S}_{2}$ & $1 / 3 \times \mathrm{B} \times \mathrm{t} 2$ & $8 / 30 \times \mathrm{B} \times \mathrm{t} 2$ & $1 / 3 \times B \times \mathrm{t} 2$ & $1 / 3 \times B \times \mathrm{t} 2$ & $1 / 3 \times B \times \mathrm{t} 2$ \\
& $=1 / 6 \times B \times T$ & $\cong 5.09 / 30 \times B \times T$ & $=1 / 4 \times B \times T$ & $=1 / 4 \times B \times T$ & $=1 / 4 \times B \times T$ \\
$\mathrm{~S}_{3}$ & 0 & $6 / 30 \times B \times \mathrm{t} 2$ & 0 & $2 / 3 \times B \times \mathrm{t} 2$ & $2 / 3 \times B \times \mathrm{t} 2$ \\
& & $\cong 3.82 / 30 \times B \times T$ & & $=1 / 2 \times B \times T$ & $=2 / 4 \times B \times T$ \\
Total & $1 / 3 \times B \times T$ & $14 / 30 \times B \times T$ & $1 / 2 \times B \times T$ & $B \times T$ & $B \times T$ \\
\hline
\end{tabular}

Similarly, to compare the available resources, the transmission time ratio of the two steps for the Hard FFR is set to $8: 14$.

Compared to a conventional relay scheme, the FFR schemes have more resources available to the BS in the first step. After providing more video layers to all users, the remaining resources of the BS in the first step of the FFR schemes are greater than in the conventional relay scheme. This remaining resource can be used to provide additional video layers to inner users. Based on the above observations, the proposed resource allocation scheme for use by all FFR methods is shown in Algorithms 1, 2, and 3. Algorithm 1 attempts to find the maximum number of video layers that can be provided to all users as a basic service and minimize resource consumption. The remaining resource is allocated to RSs in the second step by Algorithm 2, which determines the appropriate MCS of the BS and RSs to increase transmission efficiency, even if users with lower SINRs cannot receive data correctly. Algorithm 3 allocates the additional resources for the BS in the second step of the Hard FFR, Soft FFR, and Two-step power soft FFR, and determines which group should be scheduled as a higher priority and the MCS that should be employed to transmit video layers to inner users.

As shown in Algorithm 1, the proposed resource allocation scheme first determines the satisfactory video layers (denoted by $Q^{*}$ ) that all users can receive. This can be formulated as follows.

$$
\begin{aligned}
& Q^{*}= \\
& \arg \max _{Q \leq L}\left\{\sum_{x \leq Q} \sum_{\text {all } v \in V} \min _{m_{1}^{p x}, m_{2}^{* *} \in \mathrm{MCS}}\left(\left\lceil\operatorname{bit}_{\nu, x} / R\left(m_{1}^{\nu, x}\right)\right\rceil+\left\lceil\operatorname{bit}_{\nu, x} / R\left(m_{2}^{v, x}\right)\right\rceil\right)\right\}
\end{aligned}
$$

subject to

$$
\begin{aligned}
& \forall i \in N_{v}, I_{v, x}\left(i, m_{1}^{v, x}, m_{2}^{v, x}\right)=1 \\
& s_{1}^{\text {used }}=\sum_{x \leq Q^{*}} \sum_{\text {all } v \in V}\left\lceil\operatorname{bit}_{v, x} / R\left(m_{1}^{v, x}\right)\right\rceil \leq s_{1} \\
& s_{2}^{\text {used }}=\sum_{x \leq Q^{*}} \sum_{\text {all } v \in V}\left\lceil\text { bit }_{v, x} / R\left(m_{2}^{v, x}\right)\right\rceil \leq s_{2}
\end{aligned}
$$

if the Traditional FFR or the Two-step power soft FFR is used, and

$$
\begin{aligned}
& \left(\sum_{x \leq Q^{*}} \sum_{\text {all } v \in V}\left\lceil\operatorname{bit}_{v, x} / R\left(m_{1}^{v, x}\right)\right\rceil\right) / C_{1} \\
& +\left(\sum_{x \leq Q^{*}} \sum_{\text {all } v \in V}\left\lceil\operatorname{bit}_{v, x} / R\left(m_{2}^{v, x}\right)\right\rceil\right) / C_{2} \leq T
\end{aligned}
$$

if the Hard FFR or the Soft FFR is used.

Equation (1) maximizes the number of video layers $Q$ while minimizing the resource consumption, where bit $v_{v, x}$ is the bit rate of the $x$ th layer of video $\mathrm{v}$ (denoted as $v_{x}$ ). The MCS $m_{1}^{v, x}$ used by the BS in the first step and MCS $m_{2}^{v, x}$ used by the RSs in the second step are found by Equation (1). The transmission rate $R\left(m_{j}^{v, x}\right)$ is determined according to which $m_{j}^{v, x}$ is used. To transmit the video layer $v_{x}$ with the MCS $m_{j}^{v, x}$ requires at least $\left\lceil\right.$ bit $\left._{v, x} / R\left(m_{j}^{v, x}\right)\right\rceil$ resource slots, which is the ceiling value of $\operatorname{bit}_{v, x} / R\left(m_{j}^{v, x}\right)$. In other words, this is the smallest integer that is greater than or equal to bit $_{v, x} / R\left(m_{j}^{v, x}\right)$. A higher transmission rate indicates that a user needs a higher SINR to receive data correctly. $I_{v, x}\left(i, m_{1}^{v, x}, m_{2}^{v, x}\right)$ is equal to 1 if user $i$ can receive and decode video layer $v_{x}$ correctly when the $m_{1}^{v, x}$ and $m_{2}^{v, x}$ MCSs are used, and is equal to 0 otherwise. Equation (2) checks whether all users can receive the $Q *$ layers. Equations (3) and (4) check whether there are sufficient resources for the two steps, where $s_{1}^{\text {used }}$ are the resources used by the BS in the first step, $S_{1}$ are the resources available to the $B S$ in the first step, $s_{2}^{\text {used }}$ are the resources used by the RSs in the second step, and $S_{2}$ are the resources available for the RSs in the second step. Equation (5) checks the resource constraint for the Hard FFR and the Soft FFR, where $C_{1}$ are the resources available to the $\mathrm{BS}$ in the first step within a basic service time, and $C_{2}$ are the resources available to RSs in the second step within a basic service time. Therefore, the minimum resource consumption and the maximum number of video layers $Q$ for all users can be determined by Equations (1) (5).

After determining the resources required for transmitting a satisfactory number of video layers and the bit rates of the two steps, Algorithm 2 is designed to schedule any remaining resources in the second step. In 
order to maintain fairness, the cell-edge users have a higher priority for using the remaining resources in this step. The following equations compute the transmission efficiency of the video layers. After computing the transmission efficiency, the BS decides which video layers will be transmitted.

$$
\max _{m_{1}^{p, x}, m_{2}^{0, x} \in \operatorname{MCS}}\left\{\left(\sum_{i \in N_{v}} I_{\nu, x}\left(i, m_{1}^{v, x}, m_{2}^{v, x}\right)\right) /\left(\left\lceil\operatorname{bit} t_{v, x} / R\left(m_{1}^{\nu, x}\right)\right\rceil+\left\lceil b i t_{\nu, x} / R\left(m_{2}^{v, x}\right)\right\rceil\right)\right\}
$$

subject to

$$
\begin{aligned}
& s_{1}^{\text {used }}+\left\lceil\operatorname{bit}_{v, x} / R\left(m_{1}^{v, x}\right)\right\rceil \leq s_{1} \\
& s_{2}^{\text {used }}+\left\lceil\operatorname{bit}_{v, x} / R\left(m_{2}^{v, x}\right)\right\rceil \leq s_{2}
\end{aligned}
$$

if the Traditional FFR or the Two-step power soft FFR is used, and

$$
\left(s_{1}^{\text {used }}+\left\lceil\operatorname{bit}_{v, x} / R\left(m_{1}^{v, x}\right)\right\rceil\right) / C_{1}+\left(s_{2}^{\text {used }}+\left\lceil\operatorname{bit}_{v, x} / R\left(m_{2}^{v, x}\right)\right\rceil\right) / C_{2} \leq T
$$

if the Hard FFR or the Soft FFR is used.

Equation (6) is used to find the most efficient transmission rate for each step. Video layers with higher efficiency will be allocated a higher priority. In order to use resources efficiently, suitable MCSs $m_{1}^{v, x}$ and $m_{2}^{v, x}$ are found for each video group in order to increase the number of users who can successfully receive data per resource slot. In order to maintain fairness among groups, video layer $v_{x}$ would only be allocated after all lower layers $(<x)$ of all videos had already been allocated. Equations (7) and (8) check the resource consumption of transmitting video layer $v_{x}$. For the Hard FFR and the Soft FFR, the resource consumption is only checked by Equation (9).

With frequency reuse in the second step, the BS can use additional resources and the remaining resources to provide additional video layers for inner users, as shown in Algorithm 3. Similar to Equations (6) (9), the following equations are used to compute the transmission efficiency and find the most efficient transmission rate for inner users.

$$
\max _{m_{3}^{v, x} \in \mathrm{MCS}}\left\{\left(\sum_{i \in N_{v}} I_{v, x}\left(i, m_{3}^{v, x}\right)\right) /\left\lceil\mathrm{bit}_{v, x} / R\left(m_{3}^{v, x}\right)\right\rceil\right\}
$$

subject to

$$
s_{3}^{\text {used }}+\left\lceil\operatorname{bit}_{v, x} / R\left(m_{3}^{v, x}\right)\right\rceil \leq s_{1}+s_{2}+s_{3}-s_{1}^{\text {used }}-s_{2}^{\text {used }}(11)
$$

In Equation (10), $I_{v, x}\left(i, m_{3}^{v, x}\right)$ is equal to 1 if user $i$ can receive and decode video layer $v_{x}$ correctly when the $m_{3}^{v, x}$ MCS is used by the BS in the second step, and is equal to 0 otherwise. Equation (11) checks the resource consumption of transmitting video layer $v_{x}$ to inner users. $s_{3}^{\text {used }}$ denotes the resources used by the BS in the second step, and $S_{3}$ are the resources available to the $\mathrm{BS}$ in the second step.

\section{Simulation}

To evaluate the performance of the proposed schemes and the quality of the video received by users, a simulator was implemented in Java to take the path loss model, MCS, and different FFR schemes into account. The proposed scheme can be extended to wireless relay networks such as WiMAX and LTE. In this article, the simulation parameters are based on IEEE 802.16j, and parameter values suggested by the WiMAX forum in [24] are listed in Table 5. OFDMA parameters and MCSs are used according to [19], and these are listed in Tables 6 and 7. We use the COST 231 Hata [20] path loss model. In the simulation, each group provides a video to its users. Each video is encoded into five layers, and the bit rates from the first to the fifth layers are $\{32$, $32,64,128,256\}$ kbits, respectively. The system provides at least a basic layer to all users, and then provides as many video layers as possible. Each video group has 20 users on average, and each user is located at a random position within the service area. All simulation results are averaged from 1000 trials, and the network topology is regenerated for each trial. The simulation results are described as follows.

Figure 10 shows the average number of video layers received by a user. Due to the limited resources, a user can receive fewer video layers as the number of video groups increases. When there are 30 video groups in the system, there is a probability of around $10 \%$ that the conventional relay scheme cannot provide the basic layer to all users. It can be observed that when the system has more than five video groups, the Two-step power soft FFR has a $10 \%$ gain in video layers over the

\section{Table 5 System parameters}

\begin{tabular}{ll}
\hline Parameter & Value \\
\hline Operating frequency & $2.5 \mathrm{GHz}$ \\
Duplex & TDD \\
Channel bandwidth & $10 \mathrm{MHz}$ \\
Cell radius & $1200 \mathrm{~m}$ \\
BS full power & $43 \mathrm{dBm}$ \\
BS lower power & $30 \mathrm{dBm}$ \\
RS power & $40 \mathrm{dBm}$ \\
BS height & $32 \mathrm{~m}$ \\
RS height & $32 \mathrm{~m}$ \\
MS height & $1.5 \mathrm{~m}$ \\
BS antenna gain & $15 \mathrm{dBi}$ \\
RS antenna gain & $12 \mathrm{dBi}$ \\
MS antenna gain & $1 \mathrm{dBi}$ \\
MS noise figure & $7 \mathrm{~dB}$ \\
\hline
\end{tabular}


Table 6 OFDMA parameters

\begin{tabular}{ll}
\hline Parameter & Value \\
\hline Permutation mode & PUSC \\
FFT size & 1024 \\
OFDMA symbol duration & $102.9^{\circ} \mu$ \\
Frame duration & $5 \mathrm{~ms}$ \\
PUSC mode & \\
Number of data subcarriers & 720 \\
Number of pilot subcarriers & 120 \\
Number of null subcarriers & 184 \\
Number of subchannels & 30 \\
\hline
\end{tabular}

conventional relay scheme. However, as shown in Figure 11, the Soft FFR can provide more satisfactory services for the outer users. The Traditional FFR and the Twostep power soft FFR would limit the satisfactory number of video layers for all users, because the available resources of the two steps are predetermined and may be insufficient in the first or second step. For the Soft FFR, the resources available in each step can be adjusted by changing the transmission time of the two steps; thus, the Soft FFR can support more video groups when the system overloads.

Next, the average number of layers received by users located at different distances from the BS is estimated in order to verify the fairness of the FFR schemes. Better fairness implies that cell-edge users can receive more video layers. This simulation has 15 video groups in the system, and each group averages 20 users. As shown in Figure 12, the average number of layers received is estimated at intervals of $100 \mathrm{~m}$ from the BS. For example, $100200 \mathrm{~m}$ on the horizontal axis indicates users located $100200 \mathrm{~m}$ from the BS. The Soft FFR and the Two-step power soft FFR can provide more video layers for users at the cell-edge, while maintaining a good video quality for users near the BS.

Figures 13 and 14 show the average and minimum number of layers received by a user with 4,6 , and 8 RSs in the system, and a distance of 850,650 , and $650 \mathrm{~m}$, respectively, between the $\mathrm{BS}$ and the RSs. In the simulation, there are 15 and 20 video groups. As shown in Figure 13 , the average number of layers received increases

\section{Table 7 MCSs}

\begin{tabular}{cccc}
\hline \multicolumn{1}{c}{ MCS } & Required SNR (db) & Normalized capacity \\
\hline QPSK & $1 / 2$ & 5 & 1 \\
QPSK & $3 / 4$ & 8 & 1.5 \\
16-QAM & $1 / 2$ & 10.5 & 2 \\
16-QAM & $3 / 4$ & 14 & 3 \\
64-QAM & $1 / 2$ & 16 & 3 \\
64-QAM & $2 / 3$ & 18 & 4 \\
64-QAM & $3 / 4$ & 20 & 4.5 \\
\hline
\end{tabular}

as the number of RSs increases, although there is no significant increase between using six and eight RSs in the system. As shown in Figure 14, with 6 or 8 RSs and 15 groups in the system, all the schemes can provide roughly 2 video layers for the cell-edge users. With 4 RSs and 20 groups in the system, all the schemes can provide only one video layer for the cell-edge users. Moreover, as the Traditional FFR and the Soft FFR provide a better service for the cell-edge users, the number of RSs in the system has less effect on the video quality for these users.

The characteristics of the four proposed schemes can be compared in the summary in Table 8 . One observation is that the Traditional FFR, the Soft FFR, and the Two-step power soft FFR perform better than the Hard FFR. The features of these three schemes are summarized as follows. On average, the Two-step power soft FFR provides the best video quality, whereas the Traditional FFR and the Soft FFR provide better video quality for cell-edge users. The Traditional FFR provides the best video quality for outer users, while its average video quality is slightly worse than that of the Soft FFR and the Two-step power soft FFR. The Soft FFR provides the best video quality for cell-edge users, but provides fewer video layers for users who are located 400 $900 \mathrm{~m}$ from the BS than the Traditional FFR or the Two-step power soft FFR.

\section{Conclusions}

In this article, we have proposed four FFR schemes for layered video multicast over relay networks, namely Hard FFR, Traditional FFR, Soft FFR, and Two-step power soft FFR. A resource allocation scheme was also proposed for the four FFR schemes to provide as many video layers as possible to all users and provide additional video layers to inner users. Simulation results showed that cell-edge users could receive over $10 \%$ more video layers using the Traditional FFR, Soft FFR, and Two-step power soft FFR schemes than with the conventional relay scheme. These three schemes are each suited to a different distribution of users, and thus the selection of which scheme is most appropriate will depend on how the network of users is distributed. The Traditional FFR scheme performs better for users in the outer range, whereas Soft FFR performs better for celledge users, and the Two-step power soft FFR scheme gives better performance for the inner users.

\section{Algorithm 1}

1: $Q^{*}=0 / /$ Number of video layers for all users

2: for $x \leq L / /$ Index of all video layers

3: for all $v \in V / / v$ is the video played in multicast groups

4: for all available MCSs of BS $\left(m_{j}\right)$ and RSs $\left(m_{k}\right)$ 


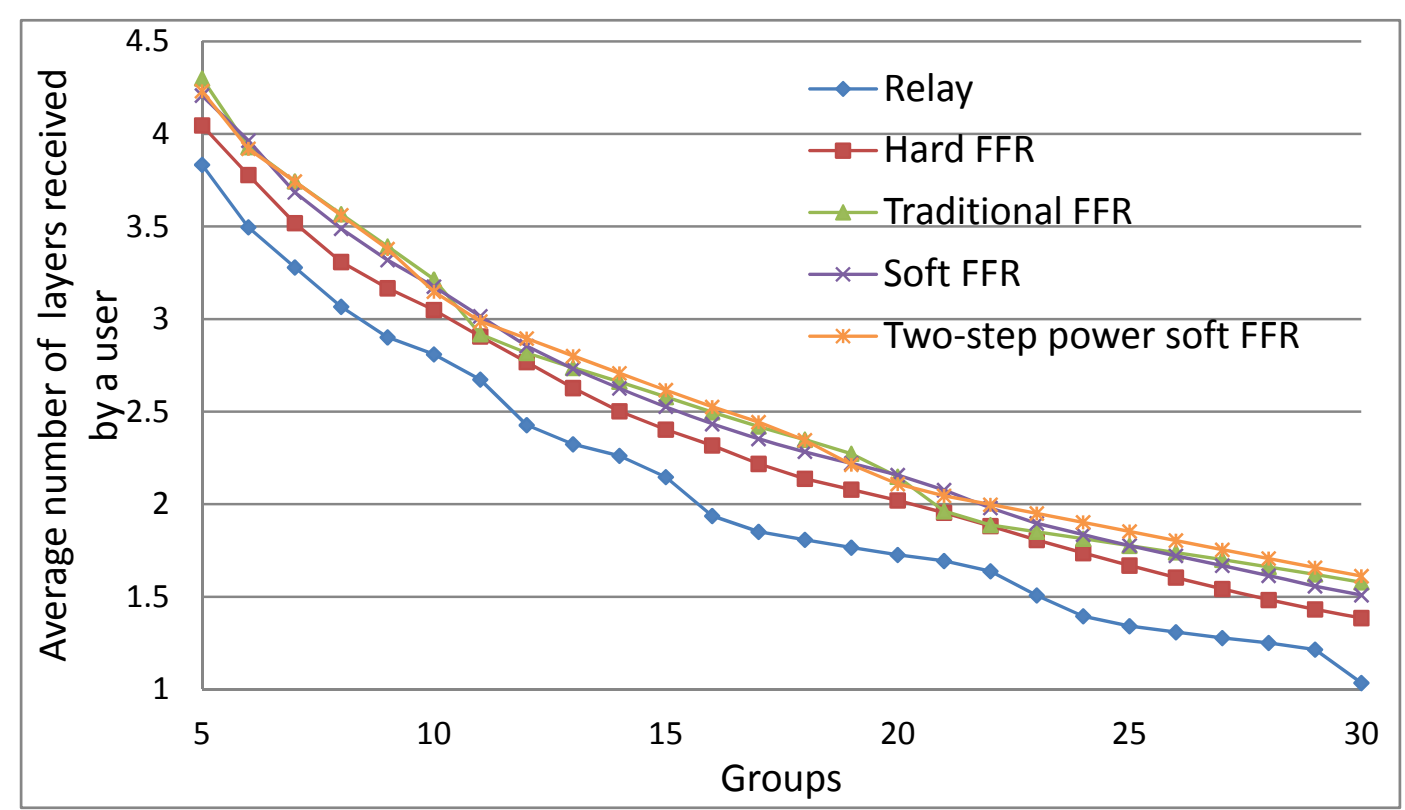

Figure 10 Average number of layers received by a user under different schemes.

5. if $\left\lceil\mathrm{bit}_{v, x} / R\left(m_{1}^{v, x}\right)\right\rceil+\left\lceil\mathrm{bit}_{v, x} / R\left(m_{2}^{v, x}\right)\right\rceil$

5: if $\begin{aligned} & >\left\lceil\operatorname{bit}_{v, x} / R\left(m_{j}\right)\right\rceil+\left\lceil\operatorname{bit}_{v, x} / R\left(m_{k}\right)\right\rceil\end{aligned}$

and $\forall i \in N_{v}, I_{v, x}\left(i, m_{j}, m_{k}\right)=1$

6: Update $m_{1}^{v, x} \leftarrow m_{j}, m_{2}^{v, x} \leftarrow m_{k}$

7: next $m_{j}, m_{k}$ pair

8: next $\mathrm{v}$

9: if satisfy Equations (3), (4) and (5)

10: schedule $v_{x}$ with $m_{1}^{v, x}, m_{2}^{v, x}$, all $v \in V$
11: $Q^{*++}$

12: else break

13: next $x$

14: $Q^{*}-$

\section{Algorithm 2}

1: for each $x, x>Q * / /$ Index of unscheduled video layers

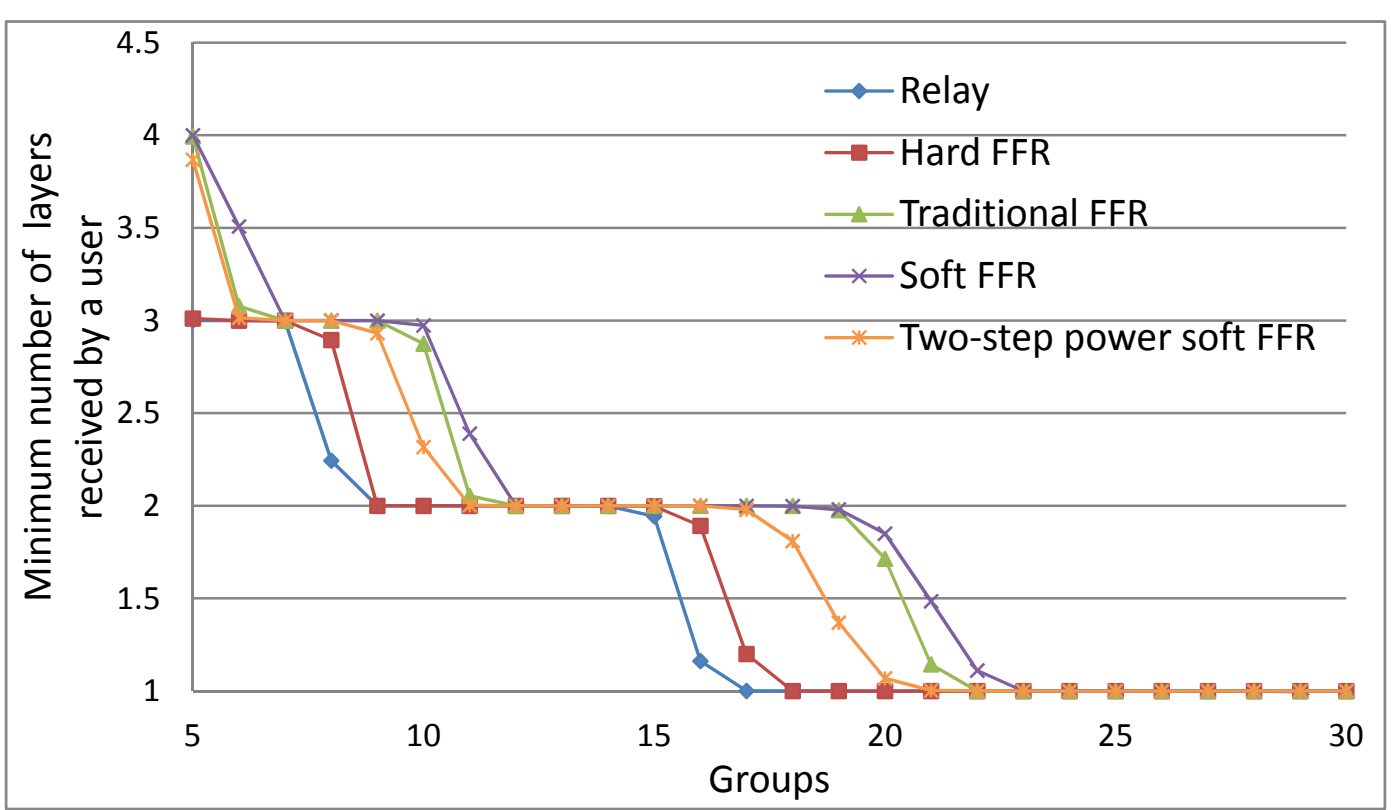

Figure 11 Minimum number of layers received by a user under different schemes. 


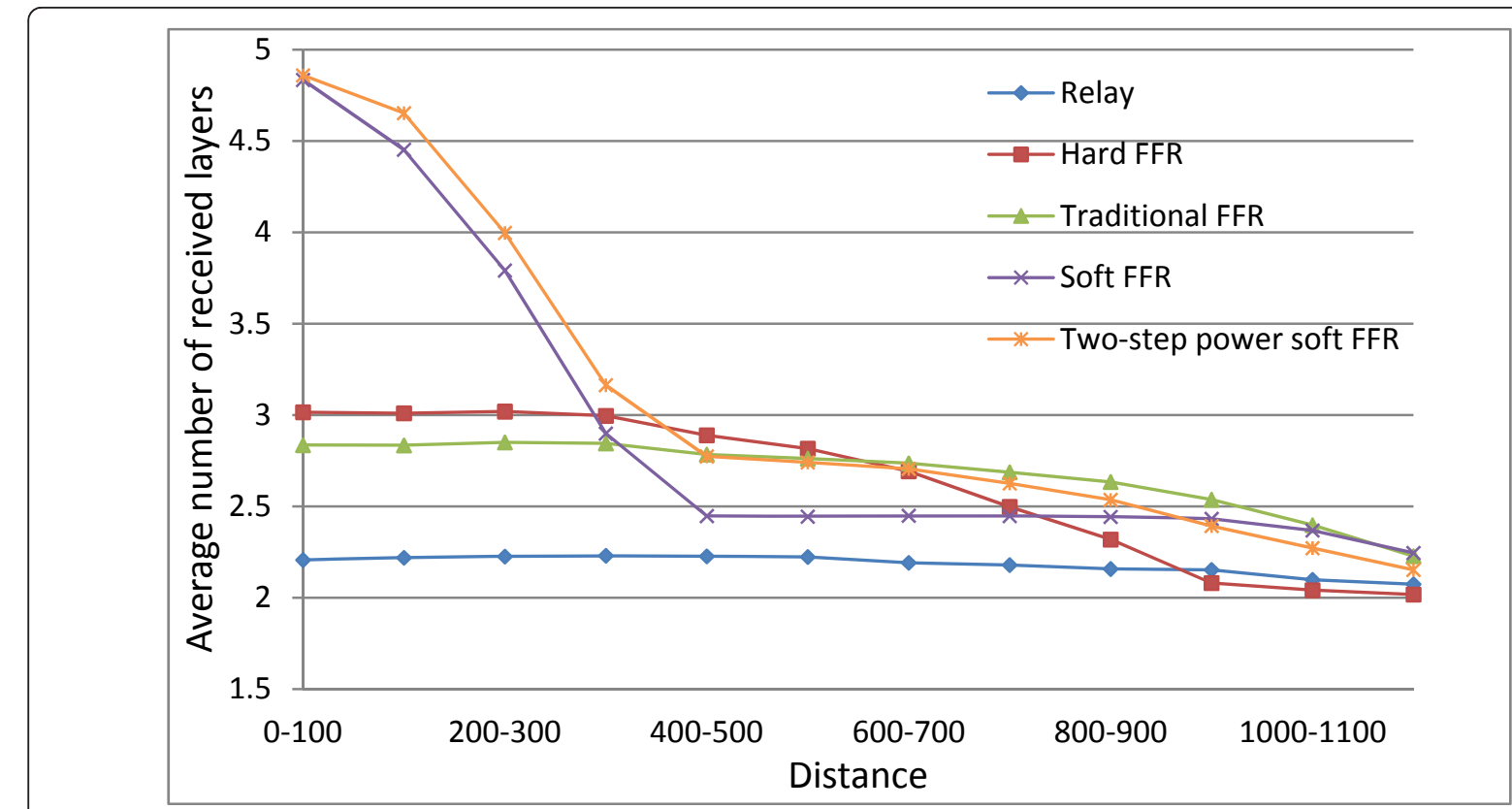

Figure 12 Average number of layers received by users vs. distance from BS (for 15 video groups in the system).

2: for all $v \in V / / v$ is the video played in multicast groups

3: for all available MCS pairs of BS $\left(m_{1}^{v, x}\right)$ and RSs $\left(m_{2}^{v, x}\right)$

//Select $m_{1}^{v, x}$ and $m_{2}^{v, x}$ to achieve the highest effiency $v_{v, x}$

4: if satisfy Equations (7), (8) and (9)
5: Compute effiency $v_{v, x}=$

$$
\left(\sum_{i \in N_{v}} I_{\nu, x}\left(i, m_{1}^{v, x}, m_{2}^{\nu, x}\right)\right) /\left(\left\lceil\operatorname{bit}_{\nu, x} / R\left(m_{1}^{\nu, x}\right)\right\rceil+\left\lceil\operatorname{bit}_{\nu, x} / R\left(m_{2}^{\nu, x}\right)\right\rceil\right)
$$

6: Store the highest effiency $v_{v, x}$ and its $m_{1}^{v, x}$ and $m_{2}^{v, x}$ 7: end if

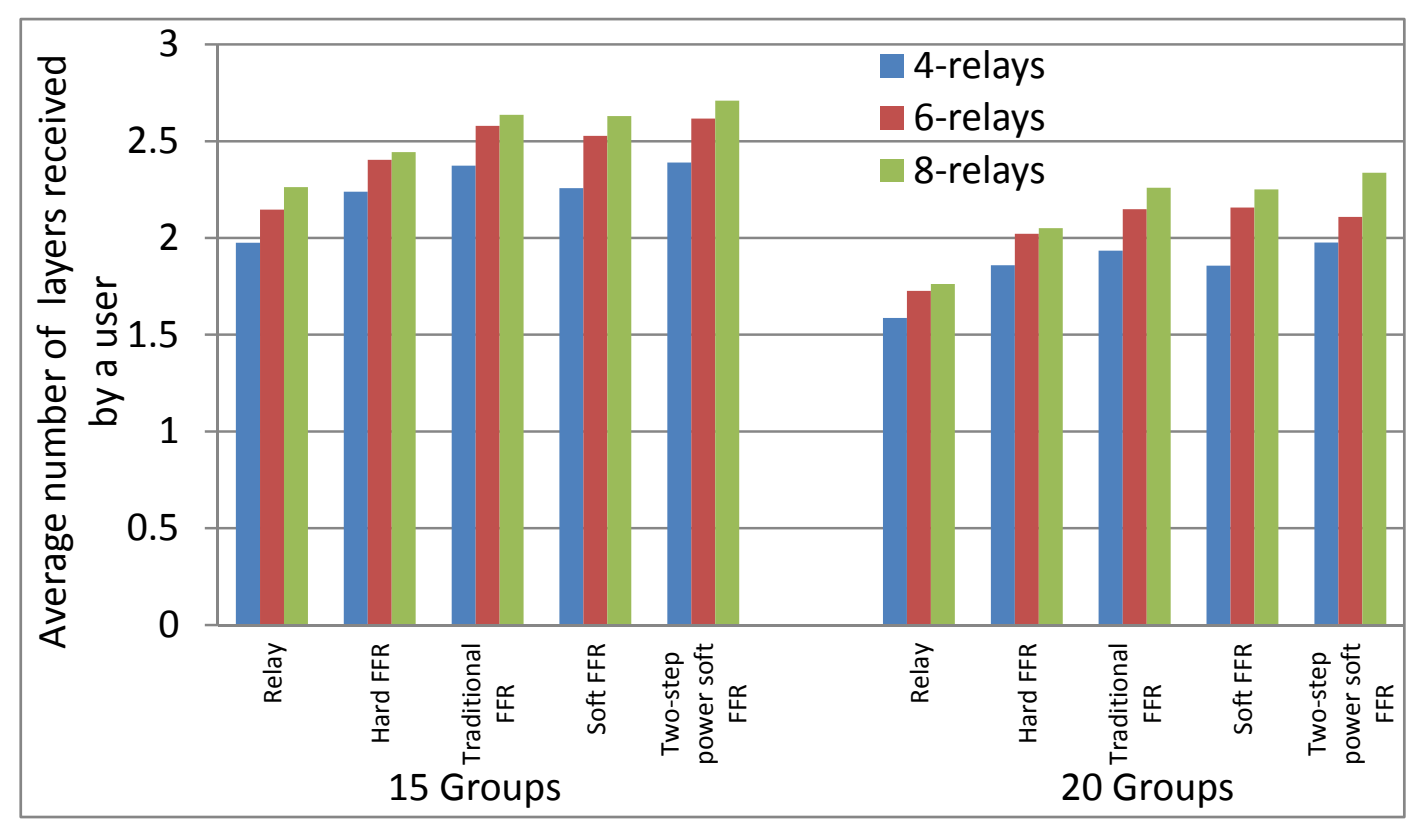

Figure 13 Average number of layers received by a user under different schemes with different numbers of RSs. 


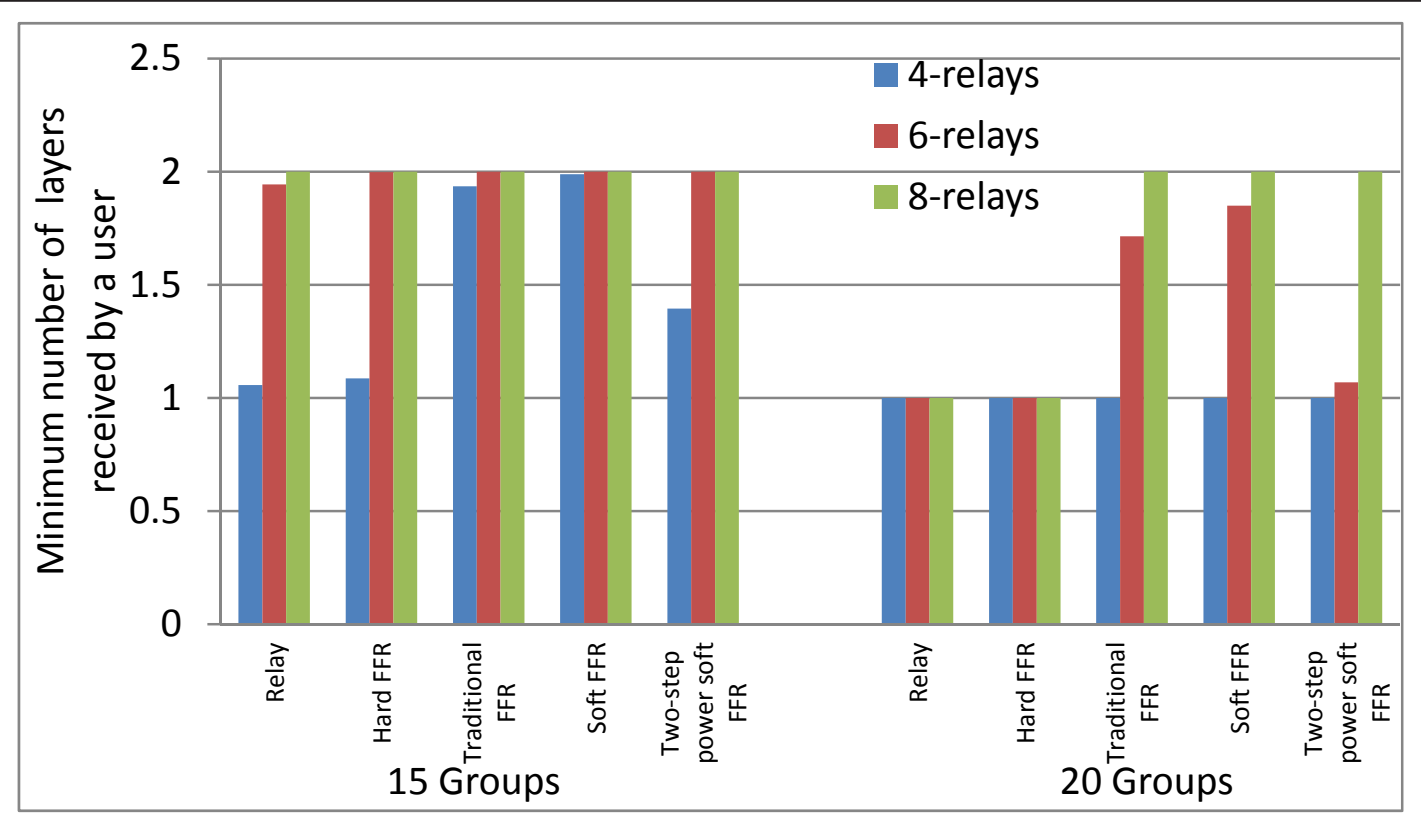

Figure 14 Minimum number of layers received by a user under different schemes with different numbers of RSs.

Table 8 Characteristics comparison of the four FFR schemes

\begin{tabular}{|c|c|c|c|}
\hline $\begin{array}{l}\text { FFR } \\
\text { scheme }\end{array}$ & $\begin{array}{l}\text { Video quality for } \\
\text { inner users }\end{array}$ & $\begin{array}{l}\text { Video quality for } \\
\text { outer users }\end{array}$ & Drawbacks \\
\hline Hard & Medium & Bad & \\
\hline Traditional & Medium & Best for outer users & Need to synchronize to neighboring cells \\
\hline Soft & High & $\begin{array}{l}\text { Best for cell-edge } \\
\text { users }\end{array}$ & Lower SINR for inner users as BS lowers its transmission power in both steps \\
\hline $\begin{array}{l}\text { Two-step } \\
\text { soft }\end{array}$ & High & Good & $\begin{array}{l}\text { Must be synchronized to neighboring cells and has a more complicated power control as the } \\
\text { BS lowers its transmission power in the second step only }\end{array}$ \\
\hline
\end{tabular}

8: next $m_{1}^{v, x}, m_{2}^{v, x}$ pairs

9: next $\mathrm{V}$

10: Sort $v_{x}$ by efficiency $v_{v, x}$ in descending order

11: for each $v_{x}, v \in V$

12: Schedule $v_{x}$ if satisfy Equations (7), (8) and (9)

13: Update $s_{1}^{\text {used }} \leftarrow s_{1}^{\text {used }}+\left\lceil\operatorname{bit}_{v, x} / R\left(m_{1}^{v, x}\right)\right\rceil$

$$
s_{2}^{\text {used }} \leftarrow s_{2}^{\text {used }}+\left\lceil\text { bit }_{v, x} / R\left(m_{2}^{v, x}\right)\right\rceil
$$

14: next $\mathrm{v}_{\mathrm{x}}$ has the highest efficiency $\mathrm{v}_{v, x}$

15: if resources are not enough break

16: $\operatorname{next} x$

\section{Algorithm 3}

1: for $x \leq L / /$ Index of unscheduled video layers

2: for all $v \in V / /$ each video belong to video multicast groups

3: for all available MCS of BS $\left(m_{3}^{v, x}\right)$

// Select $m_{3}^{v, x}$ to achieve the highest effiency $v_{v, x}$ 4: if satisfy Equation (11)
5: Compute effiency ${ }_{v, x}=$

$$
\left(\sum_{i \in N_{v}} I_{v, x}\left(i, m_{3}^{v, x}\right)\right) /\left\lceil\operatorname{bit}_{v, x} / R\left(m_{3}^{v, x}\right)\right\rceil
$$

6: Store the highest effiency ${ }_{v, x}$ and its $m_{3}^{v, x}$

7: end if

8: next $m_{3}^{v, x}$

9: next $v$

10: Sort $v_{x}$ by efficiency $y_{v, x}$ in descending order

11: for each $v_{x}$ that are not scheduled, $v \in V$

12: Schedule $v_{x}$ if satisfy Equation (11)

13: Update $s_{3}^{\text {used }} \leftarrow s_{3}^{\text {used }}+\left\lceil\operatorname{bit}_{v, x} / R\left(m_{3}^{v, x}\right)\right\rceil$

14: next $v_{x}$ has the highest efficiency ${ }_{v, x}$

15: If resources are not enough break

16: Next $x$

\section{Competing interests}

The authors declare that they have no competing interests. 
Received: 3 October 2011 Accepted: 31 May 2012

Published: 31 May 2012

\section{References}

1. Ö Alay, T Korakis, Y Wang, Layered wireless video multicast using relays. IEEE Trans Circ Syst Video. 20(8), 1095-1109 (2010). doi:10.1109/ TCSVT.2010.2056951

2. J Jin, B Li, Cooperative multicast scheduling with random network coding in WiMAX, Proceedings of the 17th International Workshop on Quality of Service, Charleston, SC, USA, 13-15 *July 2009). doi:10.1109/ IWQoS.2009.5201384

3. R Giuliano, C Monti, P Loreti, Wimax fractional frequency reuse for rural environments. IEEE Wirel Commun. 15(3), 60-65 (2008). doi:10.1109/ MWC.2008.4547524

4. G Boudreau, J Panicker, N Guo, R Chang, N Wang, S Vrzic, Interference coordination and cancellation for $4 \mathrm{G}$ networks. IEEE Commun. Mag. 47(4), 74-81 (2009). doi:10.1109/MCOM.2009.4907410

5. N Himayat, S Talwar, A Rao, R Soni, Interference management for $4 \mathrm{G}$ cellular standards. IEEE Commun Mag. 48(8), 86-92 (2010). doi:10.1109/ MCOM.2010.5534591

6. T Ali-Yahiya, H Chaouchi, Fractional frequency reuse for hierarchical resource allocation in mobile WiMAX networks. EURASIP J Wirel Commun Netw. 2010-7 (2010). Article ID 363065, doi:10.1155/2010/363065

7. J Kim, J Cho, H Shin, Layered resource allocation for video broadcasts over wireless networks. IEEE Trans Consum Electron. 54(4), 1609-1616 (2008). doi:10.1109/TCE.2008.4711209

8. Z Liu, Z Wu, P Liu, H Liu, Y Wang, Layer bargaining: multicast layered video over wireless networks. IEEE J Sel Areas Commun. 28(3), 445-455 (2010). doi:10.1109/JSAC.2010.100415

9. F Hou, LX Cai, P-H Ho, X Shen, J Zhang, A cooperative multicast scheduling scheme for multimedia services in IEEE 802.16 networks. IEEE Trans Wirel Commun. 8(3), 1508-1519 (2009). doi:10.1109/TWC.2009.080417

10. SM Elrabiei, MH Habaebi, Energy efficient cooperative communication in single frequency networks, In Proceedings of the 21st Annual IEEE International Symposium on Personal, Indoor and Mobile Radio Communications, Istanbul, Turkey, 26-29 (26-29 September 2010). doi:10.1109/PIMRC.2010.5671919

11. SW Peters, RW Heath, The future of WiMAX: multihop relaying with IEEE 802.16j. IEEE Commun Mag. 47(1), 104-111 (2009). doi:10.1109/ MCOM.2009.4752686

12. HV Zhao, W Su, Cooperative wireless multicast: performance analysis and power/location optimization. IEEE Trans Wirel Commun. 9(6), 2088-2100 (2010). doi:10.1109/TWC.2010.06.091423

13. C He, O Yang, G Wang, Performance evaluation of a WiMAX multi-hop relay system to support multicast/broadcast service, In Proceedings of the IEEE 6th International Conference on Mobile Adhoc and Sensor Systems, Macau (S.A.R.), China, 12-15 (12-15 October 2009). doi:10.1109/ MOBHOC.2009.5336937

14. P Sendín-Raña, FJ Gonzaílez-Castaño, F Gil-Castiñeira, PS RodríguezHernaíndez, Dynamic multicast groups with adaptive path selection in MMR WiMAX networks, In Proceedings of the Military Communications Conference, San Jose, CA, USA (31 October-3 November 2010). doi:10.1109/ MILCOM.2010.5680155

15. W-H Sheen, S-J Lin, C-C Huang, Downlink optimization and performance of relay-assisted cellular networks in multicell environments. IEEE Trans Veh Technol. 59(5), 2529-2542 (2010). doi:10.1109/TVT.2010.2042739

16. W-H Kuo, J-F Lee, Multicast recipient maximization in IEEE 802.16j WiMAX relay networks. IEEE Trans Veh Technol. 59(1), 335-343 (2010). doi:10.1109/ TVT.2009.2031554

17. Y-J Yu, A-C Pang, Y-C Fang, P-F Liu, Utility-based resource allocation for layer-encoded multimedia multicasting over wireless relay networks, In Proceedings of the IEEE Global Telecommunications Conference, Honolulu, Hawaii, USA, (30 November-4 December 2009). doi:10.1109/ GLOCOM.2009.5425357

18. 3GPP TS 36.213 V9.3.0, Technical Specification Group Radio Access Network; Evolved Universal Terrestrial Radio Access (E-UTRA); Physical layer procedures. Release 9 (3 October 2010)

19. IEEE Std 802.16-2009 (Revision of IEEE Std 802.16-2004), IEEE Standard for Local and metropolitan area networks Part 16: Air Interface for Broadband Wireless Access Systems. (29 May 2009) doi:10.1109/IEEESTD.2009.5062485
20. VS Abhayawardhana, IJ Wassell, D Crosby, MP Sellars, MG Brown, Comparison of empirical propagation path loss models for fixed wireless access systems, In Proceedings of the IEEE 61st Vehicular Technology Conference, Stockholm, Sweden,(30 May-1 June 2005) . doi:10.1109/ VETECS.2005.1543252

21. W Zhang, Y Li, X-G Xia, PC Ching, KB Letaief, Distributed space-frequency coding for cooperative diversity in broadband wireless ad hoc networks. IEEE Trans Wirel Commun. 7(3), 995-1003 (2008). doi: 10.1109/ TWC.2008.060717

22. $W X i e, L$ Song, $Y X u, W$ Zhang, A cooperative multicast strategy based on spatial diversity with power constraint, In Proceedings of the International Conference on Wireless Communications and Signal Processing, Suzhou, China (21-23 October 2010). doi:10.1109/WCSP.2010.5632332

23. SM Elrabiei, MH Habaebi, Energy efficient cooperative multicasting for MBS WiMAX traffic, In Proceedings of the IEEE 5th International Symposium on Wireless Pervasive Computing, Palazzo Ducale, Modena, Italy, (5-7 May 2010). doi:10.1109/ISWPC.2010.5483778

24. WiMAX Forum, Mobile WiMAX-Part I, A technical overview and performance evaluation. (2006), http://www.wimaxforum.org/technology/ downloads/Mobile_WiMAX_Part1_Overview_and_Performance.pdf

doi:10.1186/1687-1499-2012-187

Cite this article as: Lee et al.: Layered video multicast using fractional frequency reuse over wireless relay networks. EURASIP Journal on Wireless Communications and Networking 2012 2012:187.

\section{Submit your manuscript to a SpringerOpen ${ }^{\mathcal{O}}$ journal and benefit from:}

- Convenient online submission

- Rigorous peer review

- Immediate publication on acceptance

- Open access: articles freely available online

- High visibility within the field

- Retaining the copyright to your article

Submit your next manuscript at $\gg$ springeropen.com 